\title{
Expresión Morfológica del Músculo Axilopectoral. Un Estudio Anatómico Directo en una Muestra de Población Colombiana
}

\author{
Morphological Expression of the Axillary Pectoral Muscle. A Direct \\ Anatomical Study in a Sample of Colombian Population
}

Luis Ernesto Ballesteros Acuña1; Fabio Alejandro Bravo Pacheco² \& Pedro Luis Forero Porras ${ }^{3,4}$

\begin{abstract}
BALleSTEROS, A. L. E. 1; BRAVO, P. F. A. \& FORERO, P. P. L. Expresión morfológica del músculo axilopectoral. Un estudio anatómico directo en una muestra de población colombiana. Int. J. Morphol., 37(4):1262-1266, 2019.

RESUMEN: El músculo axilopectoral (MAP) es una estructura fibromuscular accesoria de la región axilar, que se relaciona con el plexo braquial, vasos y linfonodos axilares, y es reportado en los diferentes grupos poblacionales con incidencia variable. Se evaluaron un total de 106 axilas correspondientes a 53 cadáveres frescos que fueron sometidos a autopsia. El MAP se presentó en 5 regiones axilares $(4,7 \%)$, con tres casos unilaterales $(2,8 \%)$ y uno bilateral $(0,9 \%)$. La longitud total del MAP estuvo en un rango de $81,6-119,7$ $\mathrm{mm}$, mientras que su segmento tendinoso midió 13,3-28,1 mm. El espesor de su vientre muscular fue de 7,1-52 mm y del tendinoso 6,920,1 mm. En todos los casos, el MAP se originó del músculo latísimo del dorso y se insertó en el labio lateral del surco intertubercular del húmero, adyacente a la inserción del músculo pectoral mayor. Los MAP evaluados fueron inervados por el nervio toracodorsal. En dos casos, el vientre muscular del MAP cursó con trayectoria antero medial a las fibras infraclaviculares del plexo braquial, mientras que en los tres restantes, los segmentos fibrosos de inserción del MAP estuvieron relacionados con el plexo. La incidencia del MAP encontrada en el presente estudio es similar a los reportes previos realizados en cadáveres y considerablemente mayor a los reportes anatomoquirúrgicos. La importancia del MAP radica en su implicación con el síndrome de salida torácica, trombosis profunda del miembro superior y en las complicaciones en la linfadenectomía axilar.
\end{abstract}

PALABRAS CLAVE: Musculo axilopectoral; Región axilar; Plexo braquial; Linfonodos axilares.

\section{INTRODUCCIÓN}

El MAP o músculo de Langer es la variante muscular más frecuente en la región axilar (Bertone et al., 2008; Karanlik et al., 2013), reportada por Ramsey en 1795. Es una estructura fibromuscular accesoria de dimensiones variables que se extiende desde el músculo latísimo del dorso, cruza las estructuras neuromusculares de la axila y se inserta en la cara anterior del hombro (Al Maksoud et al., 2015). Ha sido reportado en población caucásica en un 3,5\% y con mayor incidencia en población mongoloide $10,8 \%$ (Taterra \& Michael, 2018). En otros grupos poblacionales se ha reportado hasta en un $7 \%$ (Turgut et al., 2005; Magee et al., 2012; Al Maksoud et al.; Upasna et al., 2015) y se ha observado esta estructura con mayor frecuencia de manera unilateral hasta un $61,6 \%$ (Taterra \& Michael). Su morfología se caracteriza por presentar una forma triangular (Chêne et al., 2007), de base posterior, que mide de 5 a $25 \mathrm{~mm}$ de grosor, una longitud de 35 - 150 mm (Turgut et al.; Chêne et al.).
Debido a su variada morfología el MAP fue clasificado por Testut (1984) en arcos axilares completos e incompletos. Más reciente, Jelev et al. (2007) lo agrupa en arco axilar superficial (grupo I) que es la forma más común y arco axilar profundo del (grupo II) que atraviesa solo una parte del haz neurovascular (Jelev et al.). En su presentación completa se extiende desde el músculo latísimo del dorso hasta compartir inserción con el tendón del músculo pectoral mayor en el margen lateral del surco intertubercular del húmero (Besana-Ciani \& Greenall, 2005; Rai et al., 2018). El tipo incompleto termina en diferentes sitios, por ejemplo, en músculos como el coracobraquial, el bíceps braquial y la porción larga del tríceps, así como el proceso coracoides (Mérida-Velasco et al., 2003; Chêne et al.; Jelev et al.; Bertone et al.). Se han reportado inclusive arcos axilares duplicados con un mismo origen y diferente inserción (Iamsaard et al., 2012).

\footnotetext{
${ }^{1}$ Departamento de Ciencias Básicas, Facultad de Salud, Universidad Industrial de Santander, Bucaramanga, Colombia.

${ }^{2}$ Facultad de Salud, Universidad Industrial de Santander, Bucaramanga, Colombia.

${ }^{3}$ Departamento de Patología, Facultad de Salud, Universidad Industrial de Santander, Bucaramanga, Colombia

${ }^{4}$ Instituto Nacional de Medicina Legal y Ciencias Forenses, Bucaramanga, Colombia.
} 
El MAP presenta una relación directa con las estructuras axilares, como el paquete neurovascular toraco dorsal, el plexo braquial, los troncos linfáticos anteriores, laterales y la vena axilar (Chêne et al.). Lo anterior exige un reconocimiento preciso de esta estructura para brindar un abordaje quirúrgico adecuado y con ello reducir el riesgo de diagnóstico inadecuado y complicaciones durante los procedimientos imagenológicos o de intervención. Teniendo en cuenta que la variabilidad del MAP se relaciona con su incidencia y sus diversas expresiones morfológicas, sumado a la escasa información sobre esta estructura en grupos poblacionales Latinoamericanos, a pesar de su gran significancia clínica, se plantea evaluar la incidencia y caracterización de la morfología de este músculo en una muestra de material cadavérico de población colombiana, que permita obtener información propia de referencia y de utilidad para aplicaciones imagenológicas y quirúrgicas de la región axilar.

\section{MATERIAL Y MÉTODO}

Este estudio descriptivo de corte transversal evaluó 106 regiones axilares de 53 individuos, a quienes se les realizo necropsia en el Instituto Nacional de Medicina legal y ciencias forenses en Bucaramanga, Colombia. Se excluyó a los sujetos con evidencia de trauma directo, cicatrices o antecedentes de intervención quirúrgica en la región axilar. El protocolo de investigación se desarrolló en estricta conformidad con las pautas éticas para la investigación médica y de salud que involucra a sujetos humanos y fue aprobado por el Comité de Ética de la Universidad Industrial de Santander.

Se realizó incisión longitudinal a lo largo de la línea media axilar, extendiéndola por la pared medial del brazo, que comprometió piel, tejido celular subcutáneo y fascia axilar. Posteriormente, se liberaron colgajos fasciocutaneos anterior y posterior que dejó expuesto los músculos pectoral mayor, menor, latísimo del dorso coracobraquial y bíceps, además del contenido neurovascular de la axila. Luego se procedió a la disección de los componentes infraclaviculares del plexo braquial y a la liberación de tejido adiposo ubicado en las superficies de los músculos descritos. Se realizó la disección cuidadosa del segmento distal del músculo latísimo del dorso hasta su inserción en el surco intertubercular del humero y se valoró la presencia del MAP, en quien se evaluó sus características cualitativas como su relación con estructuras neurovasculares de axila y se clasificaron de acuerdo a los criterios de Testut (Jelev et al.). Con micrómetro digital (Mitotuyo $®$ ) se realizaron mediciones de longitud total del músculo, así como de su vientre muscular y del segmento tendinoso. De igual manera se midió el espesor de los vientres musculares a nivel de sus tercios proximal y distal.

\section{RESULTADOS}

De 106 regiones axilares exploradas se encontró cinco estructuras accesorias que corresponden al MAP (4,7 \%). Tres especímenes cadavéricos $(2,8 \%)$ tenían MAP unilateral mientras un espécimen $(0,9 \%)$ tenía MAP bilateral. Los cinco MAP tenían una conformación completa (Fig.1). La longitud de esta estructura muscular estuvo entre 81,6 -119,6 mm con una longitud tendinosa entre 13,3-18,1 mm (Tabla I). En tres estructuras del MAP, su vientre muscular se relacionó en una trayectoria anterior con las fibras infraclaviculares del plexo braquial, mientras que en dos especímenes quien se relacionó con el plexo braquial fue su segmento tendinoso o fibroso (Figs. 2 y 3 ).

Los MAP presentaron estructuras músculo tendinosas con proporciones diferentes para cada estructura. Así, por ejemplo, en la muestra tres en la región axilar izquierda la porción tendinosa representó $24 \%$ de la longitud total de la estructura, la porción tendinosa del MAP del lado derecho equivalía al 13,9\% del total del músculo.

La conformación triangular fue observada en todos los MAP, con una base originada a partir del margen anterior del músculo latísimo del dorso de 7,1 - $52 \mathrm{~mm}$ y una altura, medida hasta la unión de la porción tendinosa de 68,3-91,6 mm.

Tabla I. Longitud y espesor del musculo axilopectoral (MAP) en una muestra de población colombiana. Expresada en milímetros.

\begin{tabular}{|c|c|c|c|c|c|c|}
\hline $\begin{array}{c}\text { Número } \\
\text { de } \\
\text { muestra }\end{array}$ & Late ralidad & Longitud total & $\begin{array}{c}\text { Espesor proximal } \\
\text { del vientre } \\
\text { muscular }\end{array}$ & $\begin{array}{c}\text { Espesor distal } \\
\text { del vientre } \\
\text { muscular }\end{array}$ & $\begin{array}{c}\text { Ancho de la } \\
\text { porción } \\
\text { tendinosa }\end{array}$ & $\begin{array}{l}\text { Longitud del } \\
\text { segmento } \\
\text { fibroso }\end{array}$ \\
\hline 10 & MAP derecho & 109,01 & 25,56 & 12,62 & 10,56 & 19,91 \\
\hline 31 & MAP derecho & 81,57 & 52,01 & 8,50 & 20,10 & 13,29 \\
\hline 38 & MAP derecho & 104,11 & 15,97 & 14,04 & 13,04 & 14,46 \\
\hline \multirow{3}{*}{46} & MAP izquierdo & 87,76 & 11,40 & 7,99 & 6,91 & 21,06 \\
\hline & MAP derecho & 119,67 & 7,13 & 5,92 & 8,34 & 28,08 \\
\hline & Rango & $81,6-119,7$ & $7,13-52,01$ & $5,92-14,04$ & $6,9-20,1$ & $13,3-28,1$ \\
\hline
\end{tabular}


BALLESTEROS, A. L. E. 1; BRAVO, P. F. A. \& FORERO, P. P. L. Expresión morfológica del músculo axilopectoral. Un estudio anatómico directo en una muestra de población colombiana. Int. J. Morphol., 37(4):1262-1266, 2019

Tabla II. Frecuencia y lado de presentación musculo axilopectoral según algunos autores.

\begin{tabular}{lllllll}
\hline \multicolumn{1}{c}{ Autores } & $\begin{array}{c}\mathrm{N}^{\circ} \text { total de } \\
\text { axilas }\end{array}$ & Map & Map unilateral & Map bilateral & $\begin{array}{c}\text { Map derecho } \\
\text { Map } \\
\text { izquierdo }\end{array}$ \\
\hline Bertone et al. & 78 & $9(11,5 \%)$ & $7(8,9 \%)$ & $1(1,28 \%)$ & $4(5,1 \%)$ & $5(6,4 \%)$ \\
Besana-Ciani \& Greenall & 46 & $3(6,5 \%)$ & $3(6,5 \%)$ & 0 & - & - \\
Mérida-Velasco et al. & 64 & $3(4,7 \%)$ & $1(1,6 \%)$ & 0 & $3(4,7 \%)$ & $1(1,6 \%)$ \\
Natsis et al. & 214 & $5(2,3 \%)$ & $5(2,3 \%)$ & 0 & $2(0,9 \%)$ & $3(1,4 \%)$ \\
Rizk \& Harbaugh & 70 & $3(4,3 \%)$ & $3(4,3 \%)$ & 0 & $1(1,4 \%)$ & $2(2,9 \%)$ \\
Presente estudio & 106 & $5(4,7 \%)$ & $3(2,8 \%)$ & $1(0,9 \%)$ & $4(3,8 \%)$ & $1(0,9 \%)$ \\
\hline
\end{tabular}

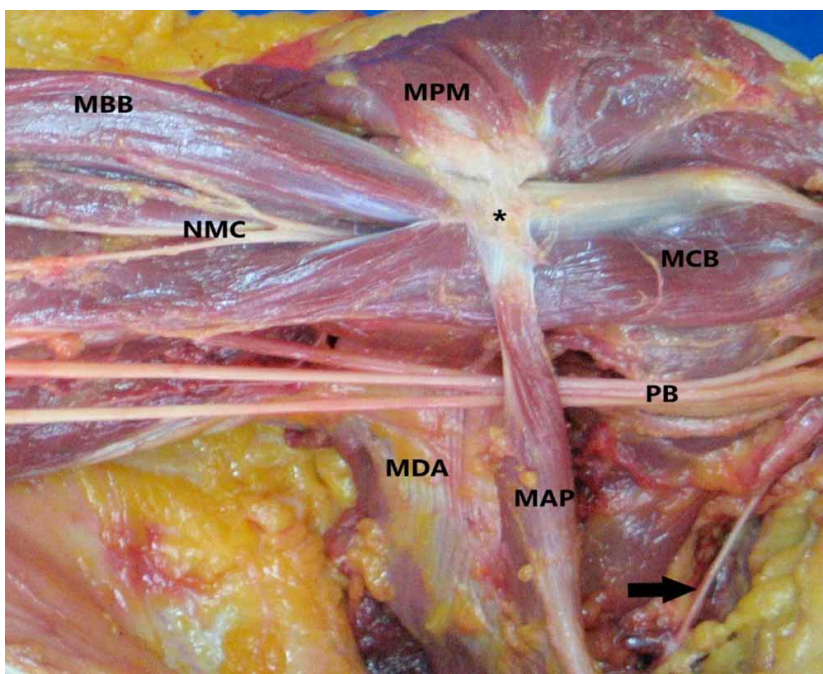

Fig. 1. Región axilar derecha. Inserción del músculo axilopectoral, cerca de la inserción del músculo pectoral mayor. MBB: Músculo bíceps braquial. MPM: Músculo pectoral mayor. MCB: Músculo coracobraquial. PB: Plexo braquial. MAP: Músculo axilopectoral. MDA: Músculo latísimo del dorso. $(\rightarrow)$ Nervio tóracodorsal. (*) Tendón del músculo axilopectoral.

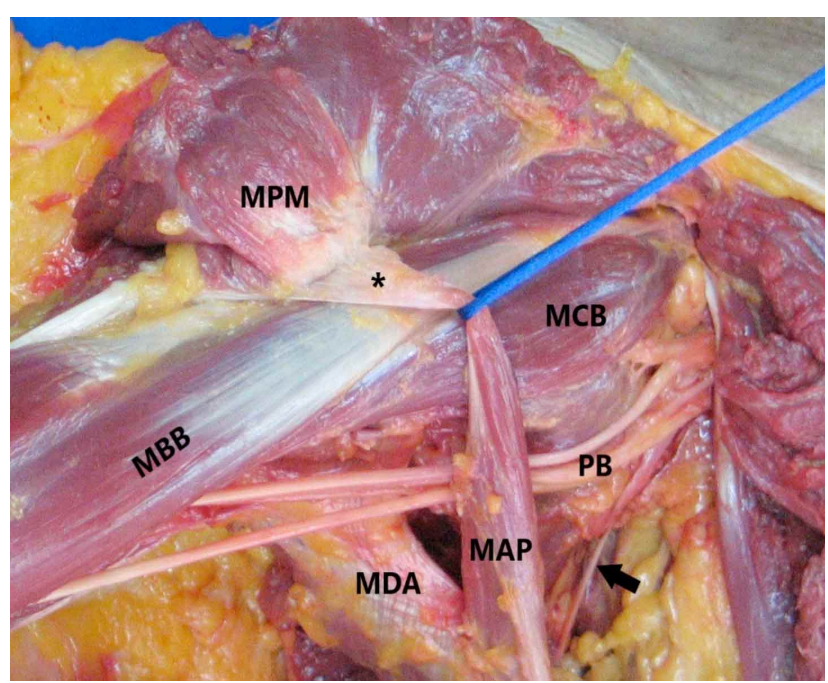

Fig. 2 Región axilar derecha. Relación del plexo braquial con el vientre muscular del músculo axilopectoral. MBB: Músculo bíceps braquial. MPM: Músculo pectoral mayor. MCB: Músculo coracobraquial. PB: Plexo braquial. MAP: Músculo axilopectoral. MDA: Músculo latísimo del dorso. $(\rightarrow)$ Nervio toracodorsal. (*) Tendón del Músculo axilopectoral.

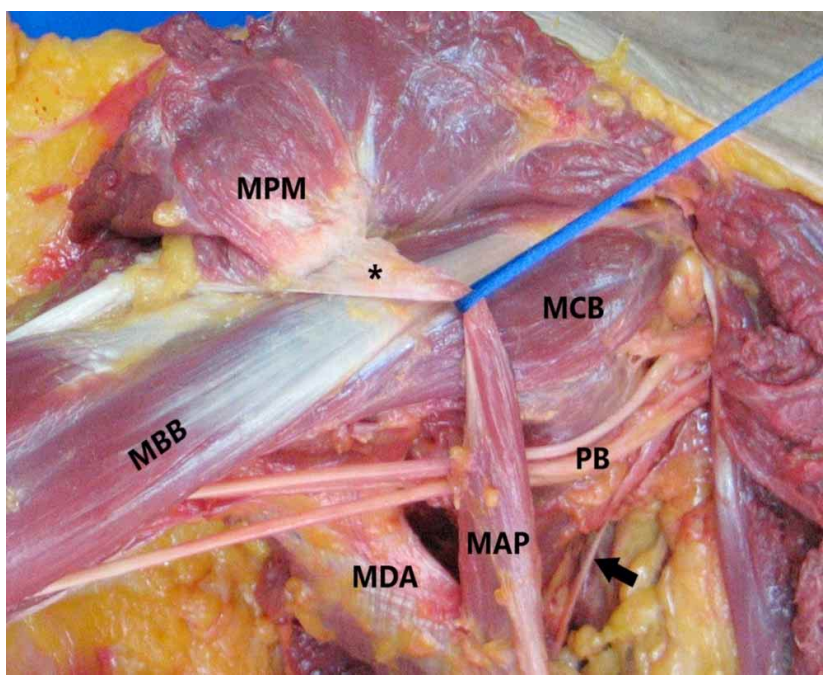

Fig. 3 Región axilar derecha. Relación del tendón del músculo axilopectoral con el plexo braquial. MBB: Músculo bíceps braquial. MPM: Músculo pectoral mayor. MCB: Músculo coracobraquial. PB: Plexo braquial. MAP: Músculo Axilopectoral. MDA: Músculo dorsal ancho. $(\rightarrow)$ Nervio toracodorsal. (*) Tendón del Músculo axilopectoral.

\section{DISCUSIÓN}

El MAP es la variación anatómica más frecuente en la región axilar. Nuestros hallazgos $(4,7 \%)$ son similares a lo reportado por (Taterra \& Michael) y ligeramente menor $(8 \%)$ a lo reportado por (Magee et al.) en material cadavérico. Se destaca la baja incidencia del MAP $(0,25-1,7 \%)$ en series con material quirúrgico (Chêne et al.; Natsis et al., 2010; Al Maksoud et al.; Koberlein \& Hoffman, 2018; Rai et al.; Taterra \& Michael). Consideramos que las diferencias entre las incidencias quirúrgicas y anatómicas de este músculo puede deberse al escaso conocimiento que de esta estructura tienen algunos cirujanos que realizan abordajes de la región axilar; Por lo que se requiere una evaluación sistémica de la región axilar y una alta sospecha clínica para detectar el MAP (Rizk \& Harbaugh, 2010).

En este estudio se encontró los MAP con conformación músculo tendinosa, correspondiendo el $81 \%$ al vientre muscular y $19 \%$ al segmento tendinoso. Se destaca que los 
estudios previos no reportan las proporciones de los vientres musculares y segmentos tendinosos del MAP; se han limitado a señalar cualitativamente si la configuración del MAP es muscular, músculo tendinosa o solamente tendinosa. Por lo cual consideramos que, en futuros estudios sobre el tema, se debe suministrar información cuantitativa sobre la conformación de esta estructura, que puede ser útil para la evaluación de las complicaciones clínicas asociadas con el MAP.

Las principales variaciones del MAP se dan en relación a su origen, inserción, tamaño y forma. En nuestro estudio encontramos que todos los MAP tenían una trayectoria completa y superficial al paquete neurovascular axilar, característica que es concordante con la mayoría de reportes previos (Mérida-Velasco et al.; Chêne et al.; Jelev et al.; Bertone et al.; Al Maksoud et al.; Koberlein \& Hoffman), con origen en el músculo latísimo del dorso e inserción en el labio lateral del surco intertubercular, adyacente a la inserción del pectoral mayor. La condición de bilateralidad del MAP hallada en nuestra serie $(0,9 \%)$ es ligeramente menor $(1,28 \%)$ al reportado por Bertone et al.. Su forma triangular fue común en nuestros hallazgos en contraste a lo aportado por Turgut et al. en quienes fue más frecuente la presentación fusiforme. La longitud total del MAP varía entre un $(81-119 \mathrm{~mm})$, el espesor de su segmento proximal (7 52) $\mathrm{mm}$ y el espesor en su segmento distal $(8-14 \mathrm{~mm})$ registrados en el presente estudio son similares a los reportados por Rai et al. (70-100 mm y 5-15 mm) y Rizk \& Harbaugh (70-90 mm y 2-3 $\mathrm{mm}$ ).

En esta serie, el MAP fue inervado por el nervio toracodorsal en concordancia con Taterra \& Michael, pero otros autores además de mencionar al toracodorsal señalan que la inervación del MAP es dada por el nervio pectoral medial (Mérida-Velasco et al.; Rai et al.). Esta diferencia en los hallazgos puede explicarse en razón a que el MAP puede insertarse en diferentes sitios como en el pectoral mayor, coracobraquial, proceso coracoides y recibir la inervación usual que tienen esos músculos vecinos. Existe controversia en cuanto lateralidad de la presentación del MAP,mientras que algunos estudios muestran una mayor incidencia de presentación en las regiones axilares izquierdas, otros coinciden con lo encontrado en nuestro estudio con una mayor presentación del MAP en la región axilar derecha (Tabla II).

La región axilar es un área de abordaje quirúrgico complicada, por la presencia estructuras neurovasculares de marcada relevancia y de los linfonodos laterales, con los que el MAP tiene una relación directa y donde se realizan procedimientos quirúrgicos de cáncer de mama, biopsia del ganglio centinela, abordajes axilares en la reparación patológica de la articulación gleno humeral o la exploración del nervio axilar (Bertone et al.). En el examen físico de la axila el MAP se puede ver como la perdida de la concavidad, masa firme o palpable que en algunos casos puede ser confundido con un linfonodo agrandado o una masa de tejido blando (Turki \& Adds, 2017; Rai et al.). En estos casos se sugiere para confirmar el diagnóstico, realizar exámenes imagenológicos complementarios como la resonancia magnética (Bertone et al.). La identificación de esta variación morfológica por parte de cirujanos y radiólogos permite reducir las posibles complicaciones de sus intervenciones.

Una de las principales complicaciones en las intervenciones quirúrgicas se da por el desconocimiento de la estructura, lo que permite una lesión accidental del plexo braquial o la arteria axilar (Upasna et al.). Por otro lado, la presencia del MAP enmascara a los linfonodos axilares posteriores, por lo que la linfadenectomia se hace más difícil y en muchos casos se requiere de una segunda intervención (Rai et al.). De igual manera, se ha reportado que el MAP puede ser causa de atrapamiento de la vena axilar generando edema de miembro superior y compresión linfática con trombosis venosa, debido a su estrecha relación con las estructuras neurovasculares y linfáticas dentro de la axila (Bertone et al.), presentándose una situación similar en la reconstrucción mamaria tardía que se realiza con el colgajo músculocutáneo del latísimo del dorso (Üçerler et al., 2005; Magee et al.). También se ha reportado que los pacientes con presencia del MAP manifiestan síntomas similares al síndrome de salida torácica por la compresión del plexo braquial por parte del MAP. Los síntomas van desde parestesias dolorosas y edema, hasta perdida de la fuerza y debilidad, síntomas que se exacerban con movimientos de abducción, rotación externa y elevación del hombro (Van Hoof et al., 2008; Rai et al.).

En conclusión, la compresión del plexo braquial, el síndrome de salida torácica, la inestabilidad del hombro, la compresión obstructiva del hombro y la sensación de masa axilar pueden ocurrir de manera secundaria a la presencia del MAP. Por otro lado, se puede deducir que quirúrgicamente el MAP puede ser el responsable de impedir una visualización adecuada de los linfonodos, lo que hace que la linfadenectomía sea incompleta y propicie iatrogenias con lesiones de las estructuras del haz neurovascular. En este contexto, es indispensable el reconocimiento de esta estructura por parte de los radiólogos lo cual conduce a su detección preoperatoria y permitirá que el equipo quirúrgico pueda realizar ajustes requeridos en los respectivos abordajes en la región axilar (Turki \& Adds). Se considera que a futuro se van a requerir estudios que relacionen los resultados preoperatorios obtenidos por ayudas diagnosticas con los hallazgos intraoperatorios que permitan reconocer el verdadero significado clínico de esta estructura. 
AGRADECIMIENTOS: Queremos expresar nuestra gratitud al Instituto Nacional de Medicina Legal y Ciencias Forenses por el suministro de las muestras anatómicas utilizadas en este estudio.

BALLESTEROS, A. L. E. 1; BRAVO, P. F. A. \& FORERO, P. P. L. Morphological expression of the axillary pectoral muscle. A direct anatomical study in a sample of Colombian population. Int. J. Morphol., 37(4):1262-1266, 2019

SUMMARY: The axillary pectoral muscle (APM) is an accessory fibromuscular structure of the axillary region. It is related to the brachial plexus, axillary vessels and lymph nodes, and is reported with variable incidence in different population groups. A total of 106 axilla were evaluated corresponding to 53 fresh cadavers. The APM was presented in 5 axillary regions (4.7\%), with three unilateral cases $(2.8 \%)$ and one bilateral $(0.9 \%)$. The total length of the APM ranged from 81.6-119.7 mm, while its tendinous segment measured 13.3-28.1 mm. Muscular belly thickness was 7.1-52 mm and the tendinous segment measured 6.9-20.1 mm. In all cases, the APM originated from the latissimus dorsi muscle and inserted into the lateral lip of humerus intertubercular sulcus, adjacent to the pectoralis major muscle insertion. The evaluated APMs were innervated by the thoracodorsal nerve. In two cases, the APM muscular belly had an anterior medial trajectory to brachial plexus fibers, while in the remaining samples, long fibrous segments of APM insertion were related to the plexus. The incidence of the APM found in the present study is similar to previous reports carried out in cadavers; it was considerably higher than previous anatomy-surgical reports. The importance of APM relies on its involvement with thoracic outlet syndrome, deep thrombosis of the upper limb and complications in axillary lymphadenectomy.

KEY WORDS: Axillopectoral muscle; Axillary region; Brachial plexus; Axillary lymph nodes.

\section{REFERENCIAS BIBLIOGRÁFICAS}

Al Maksoud, A. M.; Barsoum, A. K. \& Moneer, M. M. Langer's arch: a rare anomaly affects axillary lymphadenectomy. J. Surg. Case Rep., 2015(12):rjv159, 2015.

Bertone, V. H.; Ottone, N. E.; Lo Tartaro, M. L.; García de Quirós, N.; Dominguez, M.; Gonzalez, D.; López Bonardi, P.; Florio, S.; Lissandrello, E.; Blasi, E.; et al. The morphology and clinical importance of the axillary arch. Folia Morphol. (Warsz), 67(4):261-6, 2008.

Besana-Ciani, I. \& Greenall, M. J. Langer's axillary arch: anatomy, embryological features and surgical implications. Surgeon, 3(5):3257, 2005.

Chêne, G.; Le Bouëdec, G. \& Dauplat, J. L'arche et la sentinelle: techniques chirurgicales de la lymphadénectomie axillaire sentinelle en présence du muscle pectoro-axillaire. Gynecol. Obstet. Fertil., 35(1):25-9, 2007.

Iamsaard, S.; Uabundit, N.; Khamanarong, K.; Sripanidkulchai, K.; Chaiciwamongkol, K.; Namking, M.; Ratanasuwan, S.; Boonruangsri, P. \& Hipkaeo, W. Duplicated axillary arch muscles arising from the latissimus dorsi. Anat. Cell Biol., 45(4):288-90, 2012.

Jelev, L.; Georgiev, G. P. \& Surchev, L. Axillary arch in human: common morphology and variety. Definition of "clinical" axillary arch and its classification. Ann. Anat., 189(5):473-81, 2007.
Karanlik, H.; Fathalizadeh, A.; Ilhan, B.; Serin, K. \& Kurul, S. Axillary arch may affect axillary lymphadenectomy. Breast Care (Basel), 8(6):424-7, 2013.

Koberlein, G. C. \& Hoffman, C. Langer's axillary arch: a frequent but rarely discussed anatomical variant in the radiologic literature. Pediatr. Radiol., 48(3):433-6, 2018.

Magee, C.; Jones, C.; McIntosh, S. \& Harkin, D. W. Upper limb deep vein thrombosis due to Langer's axillary arch. J. Vasc. Surg., 55(1):234-6, 2012.

Mérida-Velasco, J. R.; Rodríguez Vázquez, J. F.; Mérida Velasco, J. A.; Sobrado Pérez, J. \& Jiménez Collado, J. Axillary arch: potential cause of neurovascular compression syndrome. Clin. Anat., 16(6):514-9, 2003.

Natsis, K.; Vlasis, K.; Totlis, T.; Paraskevas, G.; Noussios, G.; Skandalakis, P. \& Koebke, J. Abnormal muscles that may affect axillary lymphadenectomy: surgical anatomy. Breast Cancer Res. Treat., 120(1):77-82, 2010

Rai, R.; Iwanaga, J.; Loukas, M.; Oskouian, R. J. \& Tubbs, R. S. The role of the axillary arch variant in neurovascular syndrome of brachial plexus compression. Cureus, 10(6):e2875, 2018.

Rizk, E. \& Harbaugh, K. The muscular axillary arch: an anatomic study and clinical considerations. Neurosurgery, 63(4 Suppl. 2):316-9, 2008.

Taterra, D.; Henry, B. M.; Zarzecki, M. P.; Sanna, B.; Pekala, P. A.; Cirocchi, R.; Walocha, J. A.; Tubbs, R. S. \& Tomaszewski, K. A. Prevalence and anatomy of the axillary arch and its implications in surgical practice: A meta-analysis. Surgeon, 17(1):43-51, 2019.

Turgut, H. B.; Peker, T.; Gülekon, N.; Anil, A. \& Karaköse, M. Axillopectoral muscle (Langer's muscle). Clin. Anat., 18(3):220-3, 2005.

Turki, M. A. \& Adds, P. J. Langer's axillary arch: a rare variant, and prevalence among Caucasians. Folia Morphol. (Warsz), 76(3):536-9, 2017.

Üçerler, H.; Aktan Ikiz, Z. A. \& Pinan, Y. Clinical importance of the muscular arch of the axilla (axillopectoral muscle, Langer's axillary arch). Acta Chir. Belg., 105:326-8, 2005.

Upasna; Kumar, A.; Singh, B. \& Kaushal, S. Muscular variations during axillary dissection: a clinical study in fifty patients. Niger. J. Surg., 21(1):60-2, 2015.

Van Hoof, T.; Vangestel, C.; Forward, M.; Verhaeghe, B.; Van Thilborgh, L.; Plasschaert, F.; De Muynck, M.; Vanderstraeten, G. \& D'Herde, K. The impact of muscular variation on the neurodynamic test for the median nerve in a healthy population with Langer's axillary arch. $J$. Manipulative Physiol. Ther., 31(6):474-83, 2008.

\section{Dirección para correspondencia: \\ Dr Luis E. Ballesteros A. \\ Av. González valencia \# 54-25 \\ Bucaramanga \\ COLOMBIA}

Email: Iballest56@yahoo.es

Recibido : 08-03-2019

Aceptado : 17-06-2019 